\title{
BMJ Open Seroepidemiology of hepatitis A and B in the general population in Hong Kong: protocol of a cross-sectional survey using spatial sampling in a highly urbanised city
}

Chin Man Poon (D), Denise P Chan (D), Shui Shan Lee (D) , Ngai Sze Wong (D)

To cite: Poon CM, Chan DP, Lee SS, et al. Seroepidemiology of hepatitis $A$ and $B$ in the general population in Hong Kong: protocol of a crosssectional survey using spatial sampling in a highly urbanised city. BMJ Open 2021;11:e042065. doi:10.1136/ bmjopen-2020-042065

- Prepublication history and additional materials for this paper is available online. To view these files, please visit the journal online (http://dx.doi. org/10.1136/bmjopen-2020042065).

Received 24 June 2020 Revised 04 January 2021 Accepted 04 March 2021
Check for updates

(c) Author(s) (or their employer(s)) 2021. Re-use permitted under CC BY-NC. No commercial re-use. See rights and permissions. Published by BMJ.

Stanley Ho Centre for Emerging Infectious Diseases, The Chinese University of Hong Kong, Hong Kong, China

Correspondence to

Dr Ngai Sze Wong;

candy_wong@cuhk.edu.hk

\section{ABSTRACT}

Introduction Differences in immunisation policies have significantly reshaped the epidemiology of hepatitis A and $B$ in the population. Assessment of the susceptibility and transmission potential of these two types of vaccinepreventable hepatitis would enhance the capacity of public health authorities for viral hepatitis elimination. Focusing on Hong Kong, the objectives of this study comprise the determination of the population-level seroprevalence of hepatitis $A$ and $B$ and an examination of the risk factors for virus transmission and the population impacts of vaccinations.

Methods and analysis This is a cross-sectional household survey on hepatitis A and B. By using socially homogeneous building groups as sampling frame, eligible members of 1327 spatially selected households would be invited to complete a questionnaire and provide blood samples for serological testing (anti-hepatitis A virus, hepatitis B surface antigen, hepatitis B surface and core antibody). The main measures comprise a set of metrics on the prevalence of hepatitis A and B. Analysis would be conducted to examine the association of risk factors with the tested markers and describe the attitudes towards viral hepatitis vaccination.

Ethics and dissemination Ethical approval from the Joint Chinese University of Hong Kong - New Territories East Cluster Clinical Research Ethics Committee, and approval for laboratory safety from the Chinese University of Hong Kong have been obtained. The study results will be presented in scientific forums to update on the epidemiology of hepatitis A and $B$ and inform the development of new vaccination strategies in Hong Kong. Trial registration number NCT04371276.

\section{INTRODUCTION}

Viral hepatitis poses a significant public health burden worldwide, comparable to other major communicable diseases, including HIV, tuberculosis and malaria. In 2015, acute infection and sequelae of chronic infection with the hepatitis viruses led to an estimated 1.34 million deaths. ${ }^{1}$ WHO Western Pacific Region bears a disproportionally high burden
Strengths and limitations of this study

- This is a territory-wide seroprevalence study on hepatitis $A$ and $B$, the epidemiology of which has been evolving under different immunisation policies in the past decades.

- The use of socially homogeneous building groups as sampling frame provides a pragmatic approach for recruiting participants for epidemiological study in highly urbanised cities, where most people live in apartments.

- Inclusion of testing for hepatitis B surface and core antibody would support the evaluation of the susceptibility and transmission patterns of hepatitis B in the general population.

- The transmission potential of hepatitis A and B in the community would be assessed by examining the association of the risk factors with the presence of the tested immunity and infection markers.

- Long period of subject recruitment, with the possibility of self-selection bias, might have some impact on the seroprevalence estimation.

of viral hepatitis, accounting for almost $40 \%$ of the related mortality worldwide. ${ }^{2}$ As a city is in this region, the epidemiology of viral hepatitis and associated transmission potential of hepatitis viruses in Hong Kong are influenced by the nearby countries.

In Hong Kong, the annual number of reported cases of all types of acute viral hepatitis ranged between 156 and 270 in 2010 s. $^{3}$ In spite of the relatively low number of acute cases, chronic infection with hepatitis B virus (HBV) remains a significant public health problem. As shown in a recent study, the overall positivity of hepatitis B surface antigen ( $\mathrm{HBsAg}$ ) was as high as $7.8 \%$ among the general population. ${ }^{4}$ For infection with hepatitis A virus (HAV), the annual number of cases has fallen from more than 1000 in the early 1990s to less than 100 in recent 
years, probably due to improved sanitation and more hygienic environment. However, there is a pressing public health need for assessing the susceptibility to HAV in the community, given an upsurge of cases in 2015 and a recent outbreak among men who have sex with men in $2016^{56}$

Infection with HAV or HBV can cause various forms of clinical diseases, which can be prevented by vaccination. Safe and effective hepatitis A and hepatitis B vaccines have been available in Hong Kong for a long time, but the use of each was under the influence of different vaccination policies. There is no government-run programme for mass immunisation against hepatitis A. According to the current local Scientific Committee on Vaccine Preventable Diseases, Centre for Health Protection, hepatitis A vaccination is recommended for priority groups including (1) persons with chronic liver disease, (2) persons with clotting factors disorders receiving plasmaderived replacement clotting factors and (3) travellers to endemic areas. ${ }^{7}$ As shown in previous territory-wide studies, the coverage of hepatitis A vaccination in the general population was persistently low at $8.5 \%$ in 2001 and $5.9 \%$ in 2016 , respectively. ${ }^{48}$

On the other hand, a universal neonatal hepatitis B vaccination programme has been implemented in Hong Kong since 1988. Since then, all new-borns in Hong Kong would have received a birth dose in the hospital, followed with two other doses according to the schedule of the childhood immunisation programme. A series of immunisation coverage surveys has been conducted among children attending preprimary institutions every 2-3 years since 2001, showing a consistently high coverage of the third dose of hepatitis B vaccine at $99 \%$ or above. ${ }^{3}$ With the availability of HBV vaccination and the initiation of universal immunisation programme, infection with HBV in the community has been decreasing. The impacts are best illustrated by the significant drop of age-specific HBsAg prevalence among young pregnant women, first-year university students and new blood donors. $^{9-12}$

Apart from determining HBsAg prevalence, evaluation of the impact of vaccination on preventing chronic hepatitis $\mathrm{B}$ at population was usually made by a series of serosurveys involving other HBV markers, namely hepatitis B surface antibody (anti-HBs) and hepatitis B core antibody (anti-HBc) ${ }^{13}$ The presence of anti-HBs generally indicates the presence of immunity from HBV infection, acquired through either vaccination or resolved infection, whereas anti-HBc is a marker of natural HBV infection. Determining the serological profile of these three markers at population level from time to time would not only reflect any changes in epidemiology but also support the review of the effectiveness of the immunisation programme. The interpretation of such epidemiological information would also be crucial for assessing the progress towards hepatitis elimination by 2030 , a global goal targeted by WHO in the Global health sector strategy on viral hepatitis, $2016-2021 .^{14}$
Knowingly, susceptibility to HAV and HBV and their associated transmission risk in the general population vary by the differential vaccination coverage, in addition to previous virus exposure, across different age groups. Existing laboratory surveillance performed by Department of Health, Hong Kong every 5 years gives agespecific seroprevalence of anti-HAV. ${ }^{15}$ It was based on antibody testing results in around 700-1100 serum specimens during the designated year, which were obtained from convenience clinical samples of patients with conditions unrelated to hepatitis. In 2015, the seroprevalence of anti-HAV was around $50 \%$ in patients aged 40 or below, and ranged between $59 \%$ and $97 \%$ in patients aged above 40. Surveillance for the prevalence of anti-HBs was conducted in selected adult communities in Hong Kong, such as newly recruited police officers and healthcare workers, HIV/AIDS patients and persons attending postexposure management clinic. ${ }^{3}$ However, there are limited population-based studies in Hong Kong addressing the risk of transmission in the entire community, leaving the understanding of the epidemiology of hepatitis A and B incomplete. ${ }^{48}$ In addition, these past studies often carried selection bias in the study participants, who joined the study through attending a health seminar ${ }^{4}$ or an invitation on phone. ${ }^{8}$ Such sampling strategies were more likely to recruit participants of increased health consciousness, higher risk of infection and greater vulnerability to liver diseases. There would also be limited control on the diversity of socioeconomic background of the study participants recruited through these methods. A household survey using alternative sampling technique might be able to address some of these limitations and complement the assessment of the epidemiological situation.

Methodologically, there are various challenges while planning subject recruitment for a territory-wide seroprevalence study. For example, simple random sampling or systematic sampling of households in a city could be costly in listing and often impractical, as a complete frame of all households in a city may not be available. Moreover, stratified sampling might not be useful, as regards the difficulties in partitioning the entire population into disjoint and exhaustive subgroups. To get around these problems, cluster sampling could be feasible for subject recruitment by geographical areas, in view of the possibility of obtaining the list of households in a smaller spatial unit as well as administrative convenience.

Focusing on the changes in susceptibility and immunity across different age groups for hepatitis $\mathrm{A}$ and $\mathrm{B}$, two vaccine-preventable diseases, this project has been conceptualised to assess the future potential of epidemic spread of HAV and HBV in Hong Kong. Hong Kong is uniquely positioned for such a study because (1) changes in the local epidemiology for hepatitis $\mathrm{A}$ and $\mathrm{B}$ are observed in recent years; (2) vaccination against HAV and HBV infection is generally available and accessible to people in need and (3) impact of vaccination strategies and other public health interventions on these two types of viral hepatitis has not been fully evaluated. An 
assessment of the seroprevalence, in addition to associated epidemiological factors, among the general population would provide updated information for evaluating HAV and HBV transmission risk and supporting the design of future vaccination strategies.

\section{Aim and objectives}

The aim of the study is to enhance the capability of public health authorities by assessing the susceptibility and transmission potential of vaccine-preventable hepatitis, namely hepatitis A and hepatitis B, in the general population in Hong Kong. It is hypothesised that improved sanitation and immunisation programme implemented in the past three decades have significantly reshaped the epidemiology of hepatitis A and B, respectively. The change in transmission risk of hepatitis viruses in the general population shall be reviewed for informing future public health interventions targeting viral hepatitis. The specific objectives of the study are, to:

1. Determine the population-level seroprevalence of hepatitis A and hepatitis B in Hong Kong.

2. Examine the risk factors for viral hepatitis transmission, and the influences of vaccination history and intention of member of the public to get vaccinated against viral hepatitis.

\section{METHODS AND ANALYSIS}

This is a cross-sectional study on two types of vaccinepreventable hepatitis, namely hepatitis A and B. The planned start and end dates for the study are 1 June 2018 and 31 May 2021, respectively. The first 2 months of the study period would be used for study planning and preparation, including formation of study team, development of questionnaire and obtaining ethical approval. Next, subject recruitment in six rounds would last for the subsequent 18 months, so as to meet the target sample size, obtain data from the structured questionnaires and collect blood samples for serological testing. At the time point after 4 months of subject recruitment, preliminary analysis of questionnaire data and laboratory testing would be initiated and ongoing throughout the subject recruitment period. In-depth data analysis and interpretation would be performed in the next 4 months after subject recruitment is completed. Final analysis would be completed by the end of 3-year study period, when final report and manuscript for scientific journal are ready to submit.

\section{Study subjects}

All Hong Kong residents living in domestic households are eligible to join the study. However, subjects who (1) are unable to understand written Chinese or English, (2) fail to give consent for whatever reason, (3) live in institution or non-domestic housing, (4) are foreign domestic helpers or (5) are normally not residents in Hong Kong would be excluded from the study.

\section{Sample size calculation}

To estimate the population-based seroprevalence of hepatitis A and B, sample size determination for one-sample problem in estimating the population proportion was used. ${ }^{16}$ The corresponding expression for the estimated sample size $\mathrm{n}$ is as follow:

$n=z_{1-\alpha / 2}^{2} \times P \times(1-P) / d^{2}$, where $z$ represents the number of standard errors away from the mean, $P$ is the unknown prevalence of the disease, and $d$ is the precision level.

The values of $P$ were estimated by taking reference from preliminary findings of the latest study conducted in Hong Kong, which gave an overall prevalence of anti-HAV and HBsAg at $68.5 \%$ and $8.9 \%$, respectively. ${ }^{17}$ While the absolute precision is desired to be set at $2.5 \%$ with $95 \%$ level of significance, the estimated sample size for determining prevalence of hepatitis A would be $1.96^{2} \mathrm{x}(0.685)$ $\mathrm{x}(1-0.685) / 0.025^{2}=1326.3$ and that for hepatitis B would be $1.96^{2} \times(0.089) \times(1-0.089) / 0.025^{2}=498$. . While seroprevalence of hepatitis A and B is going to be determined simultaneously in this study, the minimum number of independent samples required would be 1327. Specifically, at least 1327 households should be recruited, since household is the sampling unit for this study and samples within the same household are non-independent.

\section{Enrolment of subjects}

To recruit subjects from diverse geographical locations in Hong Kong, cluster sampling is adopted (figure 1). Cluster sampling is one of the common sampling techniques used for achieving sampling efficiency in household surveys, in which clusters are virtually defined as geographical units. ${ }^{18}$ The sampling frame to be used would be Building Group, which is a demarcation system developed jointly by the Census and Statistics Department, Hong Kong and the Centamap Company Limited for disseminating data of Hong Kong population census. In 2011, there were 3223 defined Building Groups covering 41327 residential buildings. In this system, buildings are grouped in the way that each Building Group has approximately similar populations, who are socially homogeneous, in terms of geographical location, building type, building age and number of residential storeys (figure 2). Each Building Group has at least 1000 residents for ensuring protection of confidentiality of individual's identity.

With the building information available in the website of Centaline Property Agency Limited, 10 households would be randomly selected from each Building Group. While the household participation rate is estimated between $2 \%$ and $5 \%$, it is expected that the minimum number of household samples required shall be met with such number of selected households. Invitation letters stating the purposes of the study would be posted to selected households for recruitment. In the invitation letter, potential risks and benefits of participating in the study would be explained and follow-up procedures for participation would be described. For each participating household, a member with next birthday forthcoming 


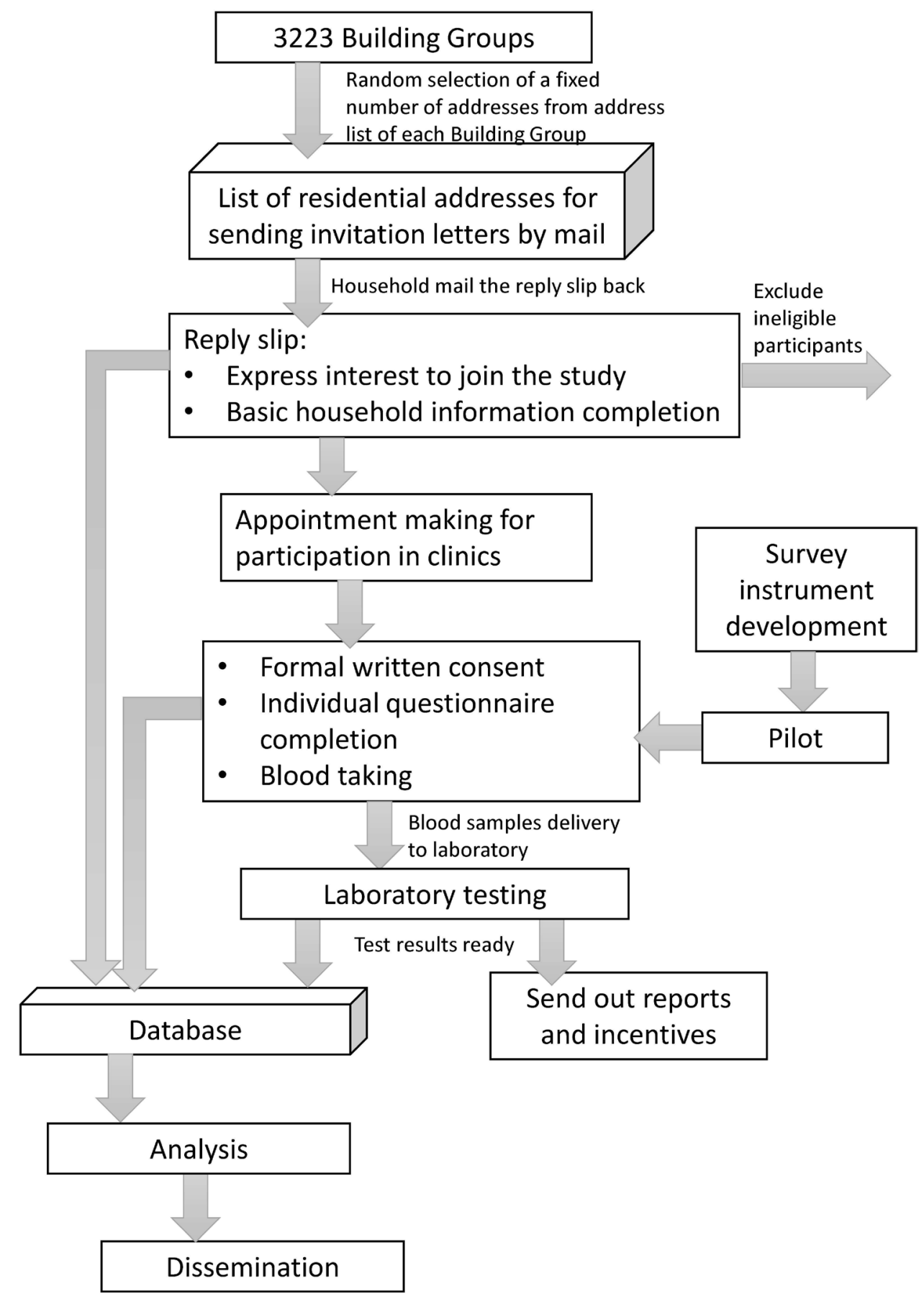

Figure 1 Study flow diagram.

would be selected as the principal participant. While infection and immunity statuses of household members are also epidemiologically important, all other eligible members in the same household would also be invited to join the study.

Participating households are invited to contact the Research Team about their willingness of participation, number of participating household members and postal address through post, phone, fax or email. On receiving consent for participation, the participants could visit the research centre or the designated clinic for blood sampling with the assistance of research staff.
To encourage participation, an incentive, in the form of catering coupon (around HKD25, US $\$ 1 \sim$ HKD 7.8), is offered to each individual who completes the entire study. A successful participation in the study includes both completion of the questionnaire and provision of blood samples.

\section{Development of questionnaire content}

A bilingual structured questionnaire is constructed in both English and Chinese languages. The questionnaire would be administered in paper format. The structured questionnaire is designed for collecting data at two 


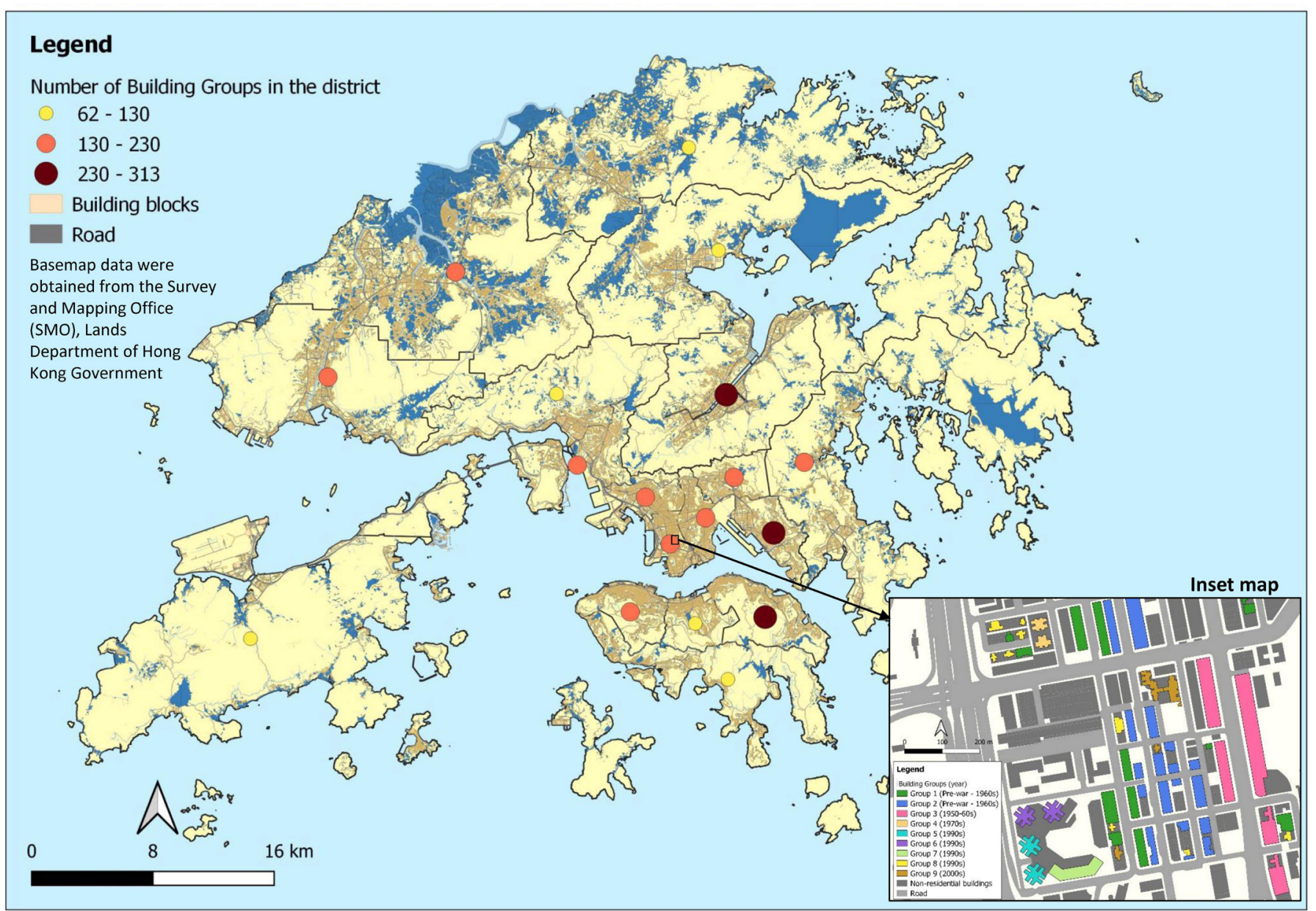

Figure 2 Distribution of building groups.

levels, namely household level and individual level. For household level data, residence district, type of housing, monthly household income, household size and household composition would be collected. In addition, the following four sections would be included in the questionnaire for collection of individual data for each participating household member (online supplemental file):

1. Demographics and socioeconomic status, including age, sex, ethnicity, place of birth, duration of staying in Hong Kong, education level, marital status and socioeconomic activity status.

2. Family history of infection of hepatitis virus and other liver diseases.

3. Risk of exposure to hepatitis virus, travel history to endemic countries, history of vaccination against viral hepatitis, and date and results of previous testing, if available, for viral hepatitis.

4. Attitudes towards and intention to receive hepatitis vaccination.

\section{Blood sampling for laboratory testing}

To determine the seroprevalence of hepatitis A and B, virus and immunity markers for these two types of viral hepatitis would be tested, respectively. For all subjects, IgG against HAV (anti-HAV IgG) and HBsAg would be tested. The presence of anti-HAV IgG would be an indicator of past infection of hepatitis A, whereas HBsAg indicates whether the subject is currently and/or chronically infected with HBV. To determine whether the subjects have been protected from hepatitis $\mathrm{B}$, additional test for anti-HBs would be conducted. Anti-HBc would also be tested to determine the prevalence of natural HBV infection. Test results with interpretation would be sent to participants.

\section{Data collation and initial analyses}

Data collected from the questionnaire survey would be input and crosschecked, and blood test results would be input for the creation of study database. For initial data exploration, standard frequency tables and matrices would be constructed. Maps showing spatial variation of the seroprevelence of hepatitis A and B by 18 districts (administrative units) would also be made using ArcGIS V.10.1 (Geographic Information System software).

By using the data obtained from the questionnaire, statistical analyses would be conducted in IBM SPSS Statistics V.25 to determine the individual and household factors associated with the positivity of anti-HAV IgG, $\mathrm{HBsAg}$, anti-HBs and anti-HBc respectively. For univariate analysis, $\chi^{2}$ test and independent sample t-test would be 
applied for categorical and continuous variables, respectively. For variables that are non-normally distributed or from small samples, non-parametric tests, say MannWhitney U test and Fisher's exact test, would be used as appropriate. Four multivariable logistic regression models would also be built, respectively, each using the status of one of these four infection or immunity markers as dependent variable. Moreover, multilevel models would be constructed for investigating the effect of household (level 2) on immunity and/or infection status of an individual (level 1). Multilevel analysis might also be applied to check if there are significant differences in social nature between sampling clusters, and adjust for their heterogeneity, if any. Differences would be classified as statistically significant when $\mathrm{p}<0.05$.

\section{Study limitations}

This study carries several limitations. First, recruitment of a large number of study participants is resourcedemanding. Instead of carrying out the subject recruitment in one go, it is expected that the target sample size would only be reached after six rounds of recruitment spanning over 18 months. Changes in hepatitis A and B seroprevalence over time in the study population, due to substantial change of risk exposure, could not be ruled out. In this case, seroprevalence in different recruitment rounds would be examined to address its variation, if any. Second, self-selection bias may still arise in this study, which adopts a geographical sampling approach. This study does not exclude known infected individuals and it is possible to attract them and their family members to join for checking their infection status. On the other hand, known infected and immune individuals may be more reluctant to join the study, while blood tests in the study would not be able to give them additional information about their health status. The impact of the selection bias on the estimation of the overall seroprevalence might be mixed. Third, the questionnaire survey involves recalling one's history of vaccination and potential risk exposure, which might introduce certain degree of information bias. Particularly, some younger participants may not be certain about their vaccination status as hepatitis $B$ vaccination is usually received during the infancy since the introduction of universal neonatal immunisation in 1988. Fourth, subjects excluded from the study include those living in institution or non-domestic housing, who are potentially linked with some common sources of hepatitis A transmission, such as residential water sources or dietary materials. The assessment of risk factors for past HAV infection and susceptibility to future HAV infection is thus limited to community-dwelling populations.

\section{ETHICS AND DISSEMINATION}

\section{Ethical consideration}

This study involves human subjects and their participation in a questionnaire survey and blood test. Foreseeable physical and psychological adverse consequences for participation in the study, such as pain and injury of using autolancet or during venesection and psychological burden of being aware of one's infection status, would be explained to potential participants. Consent will be obtained from all participants before they join the study. Participants aged below 18 will be clearly informed that their participation in the study is voluntary, and parental consent or consent from guardian would be provided in order to enrol in the study. Participants would be informed of the results, if they indicate such request in the returning questionnaire. Mechanism would be in place for participants to seek advice on the meaning and implication of test results. HBsAg-positive participants would be advised to seek hepatitis B management from medical professionals. Medical referral for disease management would be available for them.

Anonymised data would be collected. No linkage would be made between data obtained from this study and any other clinical database. Data collected would be accessed by the Principal Applicant and staff in the Research Team only. Under no circumstance would these data be available to a third party.

This study is conducted in compliance with Declaration of Helsinki. Ethical approval from the Joint Chinese University of Hong Kong-New Territories East Cluster Clinical Research Ethics Committee, and approval for laboratory safety from the Chinese University of Hong Kong have been sought.

\section{Dissemination}

The availability of vaccines continuously changes the epidemiology of hepatitis A and B in Hong Kong. The differences in vaccination coverage and previous exposure to these two hepatitis viruses across different age groups complicate their transmission dynamics. In the absence of up-to-date epidemiological information, assessment of public health burden and the potential risk of virus spread in the general population would be difficult. Furthermore, there is a clear public health need to review and optimise the current vaccination strategies for hepatitis A and B, such that healthcare resources could be allocated in a cost-effective way.

Through the generation of scientific reports and presentation in scientific forums, the findings from this study would supplement current epidemiology on viral hepatitis, and would have the following public health implications. First, new evidence would be available for explaining current epidemiological patterns of these two types of vaccine-preventable hepatitis following this study. Moreover, the findings from this study would support the evaluation of the impact of hepatitis vaccination on the susceptibility and herd immunity in the general population for informing the design of optimised immunisation programme.

From the methodological angle, the use of socially homogeneous building groups as sampling frame would be of interest to other researchers conducting studies in highly urbanised cities, like Singapore and Macau, 
where most people live in apartments. Successful execution of the study would be an example for determining prevalence of an infectious disease in an epidemiological investigation by using a geographical sampling strategy. This would also support the development of surveillance system and the design of screening strategies for infectious diseases of public health importance.

Acknowledgements Li Ka Shing Institute of Health Sciences and Stanley Ho Centre for Emerging Infectious Diseases of The Chinese University of Hong Kong are acknowledged for providing technical support in conducting the research.

Contributors CMP designed the study and wrote the protocol. SSL, NSW and DC reviewed and provided critical input to the study design and protocol. CMP wrote the first draft of the manuscript, NSW provided additional input. All authors worked collaboratively, reviewed the manuscript and made the decision to submit the final manuscript for publication.

Funding This work is supported by grants from Health and Medical Research Fund of Food and Health Bureau of the Hong Kong Special Administrative Region (No. 17160962). The authors had final responsibility for the decision to submit this manuscript for publication.

Map disclaimer The depiction of boundaries on this map does not imply the expression of any opinion whatsoever on the part of BMJ (or any member of its group) concerning the legal status of any country, territory, jurisdiction or area or of its authorities. This map is provided without any warranty of any kind, either express or implied.

Competing interests None declared.

Patient consent for publication Not required.

Provenance and peer review Not commissioned; externally peer reviewed.

Supplemental material This content has been supplied by the author(s). It has not been vetted by BMJ Publishing Group Limited (BMJ) and may not have been peer-reviewed. Any opinions or recommendations discussed are solely those of the author(s) and are not endorsed by BMJ. BMJ disclaims all liability and responsibility arising from any reliance placed on the content. Where the content includes any translated material, BMJ does not warrant the accuracy and reliability of the translations (including but not limited to local regulations, clinical guidelines, terminology, drug names and drug dosages), and is not responsible for any error and/or omissions arising from translation and adaptation or otherwise.

Open access This is an open access article distributed in accordance with the Creative Commons Attribution Non Commercial (CC BY-NC 4.0) license, which permits others to distribute, remix, adapt, build upon this work non-commercially, and license their derivative works on different terms, provided the original work is properly cited, appropriate credit is given, any changes made indicated, and the use is non-commercial. See: http://creativecommons.org/licenses/by-nc/4.0/.

\section{ORCID iDs}

Chin Man Poon http://orcid.org/0000-0003-2089-2504

Denise P Chan http://orcid.org/0000-0002-0867-1322

Shui Shan Lee http://orcid.org/0000-0003-1448-765X

Ngai Sze Wong http://orcid.org/0000-0003-3788-8114
REFERENCES

1 World Health Organization. Global hepatitis report, 2017. Available: https://apps.who.int/iris/bitstream/handle/10665/255016/ 9789241565455-eng.pdf [Accessed 4 May 2020].

2 World Health Organization. Regional office for the Western Pacific. Regional action plan for viral hepatitis in the Western Pacific 20162020: a priority action plan for awareness, surveillance, prevention and treatment of viral hepatitis in the Western Pacific Region. Available: https://apps.who.int/iris/bitstream/handle/10665/208337/ 97892906177617_eng.pdf [Accessed 4 May 2020].

3 Centre for Health Protection, Department of Health. Surveillance of viral hepatitis in Hong Kong -2018 report. Available: https://www. chp.gov.hk/files/pdf/viral_hep_sur_report_2018.pdf [Accessed on 22 Apr 2020].

4 Liu KSH, Seto WK, Lau EHY, et al. A territorywide prevalence study on blood-borne and enteric viral hepatitis in Hong Kong. $J$ Infect Dis 2019;219:1924-33.

5 Poon C. Update on hepatitis A in Hong Kong. Communicable Diseases Watch 2015;12:65-7.

6 Ho B, Wong B, Chan K. Summary on the recent upsurge of hepatitis A infection among MSM in Hong Kong. Communicable Diseases Watch 2017;14:96-7.

7 Scientific Committee on Vaccine Preventable Diseases, Centre for Health Protection, Department of Health, Hong Kong. Hepatitis A immunization in high risk groups and outbreak situations, 2006. Available: http://www.chp.gov.hk/files/pdf/sas6_hepatitis_a_ immunisation_in_high_risk_groups_and_outbreak.pdf [Accessed 4 May 2020].

8 Wong KH, Liu YM, Ng PS, et al. Epidemiology of hepatitis A and hepatitis $E$ infection and their determinants in adult Chinese community in Hong Kong. J Med Virol 2004;72:538-44.

9 Suen SS, Lao TT, Chan OK, et al. Relationship between age and prevalence of hepatitis $B$ infection in first-year university students in Hong Kong. Infection 2013;41:529-35.

10 Lao TT, Sahota DS, Suen SS, et al. Impact of neonatal hepatitis $B$ vaccination programme on age-specific prevalence of hepatitis $B$ infection in teenage mothers in Hong Kong. Epidemiol Infect 2013;141:2131-9.

11 Lao TT, Sahota DS, Law LW, et al. Age-specific prevalence of hepatitis $B$ virus infection in young pregnant women, Hong Kong special administrative region of China. Bull World Health Organ 2014;92:782-9.

12 Lin AW, Wong KH. Surveillance and response of hepatitis B virus in Hong Kong special administrative region, 1988-2014. Western Pac Surveill Response J 2016;7:24-8.

13 Cui F, Shen L, Li L, et al. Prevention of chronic hepatitis B after 3 decades of escalating vaccination policy, China. Emerg Infect Dis 2017;23:765-72.

14 World Health Organization. Global health sector strategy on viral hepatitis 2016-2021. Available: https://apps.who.int/iris/bitstream/ handle/10665/246177/WHO-HIV-2016.06-eng.pdf [Accessed 5 May 2020].

15 Centre for Health Protection, Department of Health. Seroprevalence rates of hepatitis A virus antibodies. Available: https://www.chp.gov. hk/en/statistics/data/10/641/701/3936.html [Accessed 20 May 2020].

16 Lemeshow S, Hosmer DW, Klar J. Lwanga SK: would health organisation. adequacy of sample size in health studies. Chichester: Wiley, 1990.

17 SHK L, Seto WK, Wong DK. A population-based study on the seroprevalence of viral hepatitis in Hong Kong, China. Hepatol Int 2016;10:S207-8.

18 United Nations. Designing household survey samples: practical guidelines. New York: United Nations Publication, 2008. 